\title{
Quantum phase transition of light in coupled optical cavity arrays: A renormalization group study
}

\author{
SUJIT SARKAR
}

\begin{abstract}
We study the quantum phase transition of light of a system when atom trapped in microcavities and interacting through the exchange of virtual photons. We predict the quantum phase transition between the photonic Coulomb blocked induce insulating phase and anisotropic exchange induced photonic superfluid phase in the system due to the existence of two Rabi frequency oscillations. The renormalization group equation shows explicitly that for this system there is no self-duality. The system also shows two Berezinskii-Kosterlitz-Thouless (BKT) transitions for the different physical situation of the system. The presence of single Rabi frequency oscillation in the system leads to the BKT transition where system shows the quantum phase transition from photonic metallic state to the Coulomb blocked induced insulating phase. For the other BKT transition when the z-component of exchange interaction is absent, the system shows the transition from the photonic metallic state to the photonic superfluid phase. We also predict the commensurate to incommensurate transition under the laser field detuning.
\end{abstract}

\section{Introduction}

The recent experimental success in engineering strong interaction between the photons and atoms in high quality micro-cavities opens up the possibility to use light matter system as quantum simulators for many body physics [118]. The authors of Ref. [3-5] have shown that effective spin lattice can be generated with individual atom in the microcavities that are coupled to each other via exchange of virtual photons. The two states of spin polarization are represented by the two long lived atomic levels in the system.

A Many body Hamiltonians can be created and probed in coupled cavity arrays. In our previous study, we have explained explicitly the basic physics of the formation of micro-optical cavity [5]. Atoms in the cavity are used 
for detection and also for generation of interaction between photons in the same cavity. This artificial system can act as a quantum simulator [1-6]. This micro-cavity system shows the different quantum phases and quantum phase transition (QPT) of photonic states of the system.

QPT associate with the fundamental changes that occurs in the macroscopic nature of the matter at zero temperature due to the variation of some external parameter. Quantum phase transitions are characterized by the drastic change in the ground state properties of the system driven by the quantum fluctuations $[18,19]$.

In this paper, we derive the renormalization group equation for the continuum field theoretical study of the model Hamiltonian. We predict with the existence of photonic metallic state, the anisotropic exchange induces the photonic superfluid phase and photon blocked induces the insulating phase. The transition from the photonic superfluid phase to the photon blocked induced insulating phase is the second order quantum phase transition. Whereas the transition from photonic metallic state to the photonic insulating state is the Berezinski-Kosterlitz-Thouless (BKT) transition [18, 20, 21] where the anisotropic exchange interaction is absent. The other BKT transition occurs when we consider the transition from photonic metallic state to the photonic superfluid state when the z-component interaction is absent.

The quantum state of engineering of the cavity QED system is in the state of art due to the rapid technological development in this field [1]. Therefore one can achieve the all states through the proper manipulation of laser frequencies, detuning field, Rabi frequency oscillations. In our study, the photonic metallic state is the Luttinger liquid phase of the microcavities array system. The photonic superfluid phase is originated from the anisotropic exchange interactions which discuss in Ref. [22]. To the best of our knowledge, the explicit study of the different quantum phases through the RG equations and the proposal of photonic metallic state is the first in the literature for the Cavity QED system. Now we discuss in detail about the photon blocked induce insulating phase. The photon blocked is a dressed state of strongly coupled atom-cavity system. In this phase the inhivitation of resonant absorption of a second photon if one photon is already resonantly absorbed. This phenomena was first observed by Birnbaum et al. [23]. In this studies they have predicted the photon blocked in the single atom in the cavity [23]. Here we predict this phenomena for the array of cavities, where the atoms in different cavities interacting through virtual photon emission/absorption process. 
We will see after the continuum field theoretical study that our model Hamiltonian contains two strongly relevant and mutually nonlocal perturbation over the Gaussian (critical) theory. In such a situation the strong coupling fixed point is usually determined by the most relevant perturbation whose amplitude grows up according to its Gaussian scaling dimensions and it is not much affected by the less relevant coupling terms. However, this is not the general rule if the two operators exclude each other, i.e., if the field configurations which minimize one perturbation term do not minimize the other. In this case interplay between the two competing relevant operators can produce a novel quantum phase transition through a critical point or a critical line. Therefore, we would like to study the RG equation to interpret the quantum phases of the system.

\section{Model setup and motivation}

We would like to solve the quantum phases and phase boundaries of coupled cavity arrays by establishing the quantum spin chain model of the system [35]. At first we discuss the relation between the spin operators and the atomphoton system. Our starting point is the Janes-Cummings Hamiltonian, $H=g\left(\sigma a^{\dagger}+h . c\right)$. Cavity mode represent by the bosonic operators $\left(a, a^{\dagger}\right)$ and atomic mode represent by the fermionic operator $\left(\sigma, \sigma^{\dagger}\right)$. Where $\sigma$ is the Pauli matrix which transform one excitation from the radiation field to the atomic field. Therefore, one can write

$$
\sigma^{\dagger}=\left(\begin{array}{ll}
0 & 1 \\
0 & 0
\end{array}\right)
$$

similarly one can write for $\sigma^{-}$with $<1 \mid=(1,0)$ and $<0 \mid=(0,1)$. The excitation in this system only transfer between atom and photon in the cavity. For a fixed number $(n)$ of total excitation, one can express the manifold: $H_{n}=\{|0, n>| 1,, n-1>\}$ provided $n \geq 1$. Here $\mid 0, n>$ and $\mid 1, n-1>$ are represent atom in the ground state with $n$ photon and excited states of the atom with $(n-1)$ photon respectively. We consider the initial state $\mid e, n-1>$, we obtain the state $\mid g, n>$ by the following operation, $\mid g, n>=$ $\sigma a^{+} \mid e, n-1>$. Therefore, we may write the following relation based on the conservation of the number of excitation. $\sigma^{\dagger} a\left|e, n-1>=0=\sigma a^{\dagger}\right| g, n>$ and $\sigma^{\dagger} a|g, n>=| e, n-1>$. These relations are nothing but the properties of spin operators acting on the spinors in the $\mathrm{z}$ basis.

Micro-cavities of a photonic crystal are coupled through the exchange of photons. Each cavity consists of one atom with three levels in the energy 
spectrum, two of them are long lived and represent two spin states of the system and the other represent excited states (Figs. 1 and 2 of Ref. [3, 4]). Externally applied laser and cavity modes couple to each atom of the cavity. It may induce the Raman transition between these two long lived energy levels. Under a suitable detuning between the laser and the cavity modes, virtual photons are created in the cavity which mediate interactions with another atom in a neighboring cavity. One can eliminate the excited states by choosing the appropriate detuning between the applied laser and cavity modes. Then one can achieve only two states per atom in the long lived state and the system can be described by a spin-1/2 Hamiltonian $[3,4]$.

The Hamiltonian of the system consists of three parts:

$$
H=H_{A}+H_{C}+H_{A C}
$$

Hamiltonians are the following

$$
H_{A}=\sum_{j=1}^{N} \omega_{e}\left|e_{j}><e_{j}\right|+\omega_{a b}\left|b_{j}><b_{j}\right|
$$

where $j$ is the cavity index. $\omega_{a b}$ and $\omega_{e}$ are the energies of the state $\mid b>$ and the excited state respectively. The energy level of state $\mid a>$ is set as zero. $|a\rangle$ and $\mid b>$ are the two stable state of a atom in the cavity and $\mid e>$ is the excited state of that atom in the same cavity. The following Hamiltonian describes photons in the cavity,

$$
H_{C}=\omega_{C} \sum_{j=1}^{N} a_{j}^{\dagger} a_{j}+J_{C} \sum_{j=1}^{N}\left(a_{j}^{\dagger} a_{j+1}+h . c\right)
$$

where $a_{j}^{\dagger}\left(a_{j}\right)$ is the photon creation (annihilation) operator for the photon field in the $j$ 'th cavity, $\omega_{C}$ is the energy of photons and $J_{C}$ is the tunneling rate of photons between neighboring cavities. Interaction between the atoms and photons and also by the driving lasers are described by

$$
H_{A C}=\sum_{j=1}^{N}\left[\left(\frac{\Omega_{a}}{2} e^{-i \omega_{a} t}+g_{a} a_{j}\right)\left|e_{j}><a_{j}\right|+h . c\right]+[a \leftrightarrow b] .
$$

Here $g_{a}$ and $g_{b}$ are the couplings of the cavity mode for the transition from the energy states $\mid a>$ and $\mid b>$ to the excited state. $\Omega_{a}$ and $\Omega_{b}$ are the Rabi frequencies of the lasers with frequencies $\omega_{a}$ and $\omega_{b}$ respectively. 
The authors of Ref. [3, 4] have derived an effective spin model by considering the following physical processes: A virtual process regarding emission and absorption of photons between the two stable states of neghbior cavity yields the resulting effective Hamiltonian as

$$
H_{x y}=\sum_{j=1}^{N} B{\sigma_{j}}^{z}+\sum_{j=1}^{N}\left(\frac{J_{1}}{2}{\sigma_{j}}^{\dagger} \sigma_{j+1}{ }^{-}+\frac{J_{2}}{2}{\sigma_{j}}^{-} \sigma_{j+1}{ }^{-}+h . c\right)
$$

When $J_{2}$ is real then this Hamiltonian reduces to the XY model. Where $\sigma_{j}{ }^{z}=\left|b_{j}><b_{j}\right|-\left|a_{j}><a_{j}\right|, \sigma_{j}^{+}=\left|b_{j}><a_{j}\right|, \sigma_{j}^{-}=\left|a_{j}><b_{j}\right|$

$$
\begin{aligned}
H_{x y}= & \sum_{i=1}^{N}\left(B{\sigma_{i}}^{z}+J_{1}\left(\sigma_{i}^{x} \sigma_{i+1}^{x}+\sigma_{i}^{y} \sigma_{i+1}^{y}\right)\right. \\
& \left.+J_{2}\left(\sigma_{i}^{x} \sigma_{i+1}^{x}-\sigma_{i}^{y} \sigma_{i+1}^{y}\right)\right) \\
= & \sum_{i=1}^{N} B\left(\sigma_{i}^{z}+J_{x} \sigma_{i}^{x} \sigma_{i+1}^{x}+J_{y} \sigma_{i}^{y} \sigma_{i+1}^{y}\right) .
\end{aligned}
$$

with $J_{x}=\left(J_{1}+J_{2}\right)$ and $J_{y}=\left(J_{1}-J_{2}\right)$.

Here we present the analytical expression of $B, J_{1}$ and $J_{2}$ in terms of different physical parameters of the system. We follow the references $[3,24]$.

$$
\begin{aligned}
B= & \frac{\delta_{1}}{2}-\beta \\
\beta= & \frac{1}{2}\left[\frac { | \Omega _ { b } | ^ { 2 } } { 4 \Delta _ { b } } \left(\Delta_{b}-\frac{\left|\Omega_{b}\right|^{2}}{4 \Delta_{b}}-\frac{\left|\Omega_{b}\right|^{2}}{4\left(\Delta_{a}-\Delta_{b}\right)}\right.\right. \\
& \left.-\gamma_{b} g_{b}^{2}-\gamma_{1} g_{a}^{2}+\gamma_{1}{ }^{2} \frac{g_{a}^{4}}{\Delta_{b}}-(a \leftrightarrow b)\right] \\
J_{1}= & \frac{\gamma_{2}}{4}\left(\frac{\left|\Omega_{a}\right|^{2} g_{b}{ }^{2}}{\Delta_{a}{ }^{2}}+\frac{\left|\Omega_{b}\right|^{2} g_{a}{ }^{2}}{\Delta_{b}{ }^{2}}\right) \\
J_{2}= & \frac{\gamma_{2}}{2}\left(\frac{\Omega_{a} \Omega_{b} g_{a} g_{b}}{\Delta_{a} \Delta_{b}}\right) .
\end{aligned}
$$

where

$$
\gamma_{a, b}=\frac{1}{N} \sum_{k} \frac{1}{\omega_{a, b}-\omega_{k}}, \quad \gamma_{1}=\frac{1}{N} \sum_{k} \frac{1}{\left(\omega_{a}+\omega_{b}\right) / 2-\omega_{k}}
$$




$$
\begin{aligned}
& \gamma_{2}=\frac{1}{N} \sum_{k} \frac{e^{i k}}{\left(\omega_{a}+\omega_{b}\right) / 2-\omega_{k}}, \quad \delta_{1}=\omega_{a b}-\left(\omega_{a}-\omega_{b}\right) / 2, \\
& \Delta_{a}=\omega_{e}-\omega_{a}, \quad \Delta_{b}=\omega_{e}-\omega_{a}-\left(\omega_{a b}-\delta_{1}\right), \\
& \delta_{a}{ }^{k}=\omega_{e}-\omega_{k}, \quad \delta_{b}{ }^{k}=\omega_{e}-\omega_{k}-\left(\omega_{a b}-\delta_{1}\right),
\end{aligned}
$$

$g_{a}$ and $g_{b}$ are the couplings of respective transition to the cavity mode, $\Omega_{a}$ and $\Omega_{b}$ are the Rabi frequency of laser with frequency $\omega_{a}$ and $\omega_{b}$.

Here we discuss very briefly about an effective $z$-component of interactions $\left(\sigma_{i}{ }^{z} \sigma_{i+1}{ }^{z}\right)$ in such a system. The authors of Ref. [3, 4] have proposed the same atomic level configuration but having only one laser of frequency $\omega$ that mediates the atom-atom coupling through virtual photons. Another laser field with frequency $\nu$ is used to tune the effective magnetic field. In this case the Hamiltonian $H_{A C}$ changes but the Hamiltonians $H_{A}$ and $H_{C}$ are the same.

$$
H_{A C}=\sum_{j=1}^{N}\left[\left(\frac{\Omega}{2} e^{-i \omega t}+\frac{\Lambda}{2} e^{-i \nu_{a} t} g_{a} a_{j}\right)\left|e_{j}><a_{j}\right|+h . c\right]+[a \leftrightarrow b]
$$

Here, $\Omega_{a}$ and $\Omega_{b}$ are the Rabi frequencies of the driving laser with frequency $\omega$ on transition $|a>\rightarrow| e>,|b>\rightarrow| e>$, whereas $\Lambda_{a}$ and $\Lambda_{b}$ are the driving laser with frequency $\nu$ on transition $|a>\rightarrow| e>,|b>\rightarrow| e>$. One can eliminate adiabatically the excited atomic levels and photons by considering the interaction picture with respect to $H_{0}=H_{A}+H_{C}[6,7]$. They have considered the detuning parameter in such a way that the Raman transitions between two level are suppressed and also chosen the parameter in such a way that the dominant two-photon processes are those that involve one laser photon and one cavity photon but the atom makes no transition between levels a and b. Whenever two atoms exchange a virtual photon both of them experience a Stark shift and play the role of an effective $\sigma^{z} \sigma^{z}$ interaction [3-5]. Then the effective Hamiltonian reduces to

$$
H_{z z}=\sum_{j=1}^{N}\left(B_{z} \sigma_{j}^{z}+J_{z} \sigma_{j}^{z} \sigma_{j+1}^{z}\right)
$$

These two parameters can be tuned independently by varying the laser frequencies. Finally, they have obtained an effective model by combining Hamiltonians $H_{x y}$ and $H_{z z}$ by using Suzuki-Trotter formalism. The effective Hamiltonian simulated by this procedure is 


$$
H_{\text {spin }}=\sum_{j=1}^{N}\left(B_{t o t} \sigma_{j}^{z}+\sum_{\alpha=x, y, z} J_{\alpha} \sigma_{j}^{\alpha} \sigma_{j+1}^{\alpha}\right)
$$

where $B_{t o t}=B+B_{z}$. It has been shown in Ref. [4] that $J_{y}$ is less than $J_{x}$. From the analytical expressions of $J_{x}$ and $J_{y}$, it is clear that the magnitudes of $J_{1}$ and $J_{2}$ are different. The result of numerical simulations trigger us also to define a model to study the quantum phases of this system. In the next section, we present the RG study of this model Hamiltonian to extract quantum phases and transitions between them.

Here we present the analytical expression of $B_{t o t}, J_{z}$ in terms of different physical parameters of the system. We follow the references $[3,5,24]$.

$$
\begin{aligned}
J_{z}= & \gamma_{2}\left|\frac{\Omega_{b}{ }^{*} g_{b}}{4 \Delta_{b}}-\frac{\Omega_{a}^{*} g_{a}}{4 \Delta_{a}}\right|^{2} \\
B_{t o t}= & -\frac{1}{2}\left[\frac{\left|\Lambda_{b}\right|^{2}}{16 \tilde{\Delta}_{b}^{2}}\left(4 \tilde{\Delta_{b}}-\frac{\left|\Lambda_{a}\right|^{2}}{4\left(\tilde{\Delta}_{a}-\tilde{\Delta}_{b}\right)}-\frac{\left|\Lambda_{b}\right|^{2}}{\tilde{\Delta_{b}}}-\beta_{2}\right)-\beta_{3}\right] . \\
\beta_{2}= & \sum_{j=a, b} \frac{\left|\Omega_{j}\right|^{2}}{4\left(\Delta_{j}-\tilde{\left.\Delta_{b}\right)}\right.} \cdot 4 \tilde{\gamma}_{j b} g_{j}^{2} \\
\beta_{3}= & {\left[\frac { | \Omega _ { b } | ^ { 2 } } { 1 6 \Delta _ { b } { } ^ { 2 } } \left(4 \Delta_{b}-\frac{\left|\Omega_{a}\right|^{2}}{4 \Delta_{b}}-\frac{\left|\Omega_{b}\right|^{2}}{4\left(\Delta_{a}-\Delta_{b}\right)}\right.\right.} \\
& -\frac{\left|\Omega_{b}\right|^{2}}{\Delta_{b}}-\sum_{j=a, b} \frac{\left|\Lambda_{j}\right|^{2}}{\left.4\left(\Delta_{j}-\tilde{\left.\Delta_{b}\right)} \cdot 4 \gamma_{j b} g_{j}^{2}\right)+\gamma_{b b}{ }^{2} \frac{g_{b}{ }^{4}}{\Delta_{b}}-(a \leftrightarrow b)\right]}
\end{aligned}
$$

Here

$$
\begin{aligned}
& \gamma_{1}=\frac{1}{N} \sum_{k} \frac{1}{\omega-\omega_{k}}, \quad \gamma_{2}=\frac{1}{N} \sum_{k} \frac{e^{i k}}{\omega-\omega_{k}}, \\
& \gamma_{a a}=\gamma_{b b}=\frac{1}{N} \sum_{k} \frac{1}{\omega-\omega_{k}}, \quad \gamma_{a b}=\gamma_{b a}=\frac{1}{N} \sum_{k} \frac{1}{\omega \pm \omega_{a b}-\omega_{k}} \\
& \tilde{\gamma}_{a b}=\tilde{\gamma}_{b a}=\frac{1}{N} \sum_{k} \frac{1}{\nu \pm \omega_{a b}-\omega_{k}}, \quad \tilde{\gamma}_{a a}=\tilde{\gamma}_{b b}=\frac{1}{N} \sum_{k} \frac{1}{\nu-\omega_{k}}
\end{aligned}
$$




\section{Analytical derivation and analysis of RG equations}

To study the different quantum phases of the system described by the Hamiltonian (Eq. 13), we express this Hamiltonian in more explicit way,

$$
H_{2}=\sum_{n}\left[(1+a) S_{n}^{x} S_{n+1}^{x}+(1-a) S_{n}^{y} S_{n+1}^{y}+\Delta S_{n}^{z} S_{n+1}^{z}+h S_{n}^{z}\right]
$$

where $S_{n}^{\alpha}$ are the spin- $1 / 2$ operators. We assume that the $X Y$ anisotropy $a$ and the $z z$ coupling $\Delta$ satisfy the condition $-1 \leq \Delta \leq 1$, and $0<a \leq 1$ and magnetic field strength is $h \geq 0$. The parameters correspondence between the micro cavities and spin chain are the following, $h \sim B_{t o t}, \Delta=J_{z}, J_{1}=1$ and $J_{2}=a$. The $X Y$ anisotropy breaks the in plane rotational symmetry. The study of the quantum phases from the perspective of quantum spin system and magnetism is not entirely a new one [25-27]. Here our main aim is to study the quantum phases of microcavities array through the RG analysis of this model Hamiltonian.

Spin operators can be recast in terms of spinless fermions through JordanWigner transformation and then finally one can express the spinless fermions in terms of bosonic fields [20]. We recast the spinless fermions operators in terms of field operators by this relation. $\psi(x)=\left[e^{i k_{F} x} \psi_{R}(x)+e^{-i k_{F} x} \psi_{L}(x)\right]$, where $\psi_{R}(x)$ and $\psi_{L}(x)$ describe the second-quantized fields of right- and the left-moving fermions respectively, and $k_{F}$ is the Fermi wave vector. We express the fermionic fields in terms of bosonic field by the relation $\psi_{r}(x)=\frac{U_{r}}{\sqrt{2 \pi \alpha}} e^{-i(r \phi(x)-\theta(x))}$, where $r$ denotes the chirality of the fermionic fields, right (1) or left movers (-1). The operators $U_{r}$ is the Klein factor to preserve the anti-commutivity of fermions. $\phi$ field corresponds to the quantum fluctuations (bosonic) of spin and $\theta$ is the dual field of $\phi$. They are related by the relations $\phi_{R}=\theta-\phi$ and $\phi_{L}=\theta+\phi$. Hamiltonian $H_{0}=\frac{v}{2} \int d x\left[\left(\partial_{x} \theta\right)^{2}+\left(\partial_{x} \phi\right)^{2}\right]$ is non-interacting part of $H_{X Y Z}$. Here $v$ is the velocity of the low-energy excitations. It is one of the Luttinger liquid parameters and the other is $K$, which is related to $\Delta$ by $[20,26]$

$$
K=\frac{\pi}{\pi+2 \sin ^{-1}(\Delta)} .
$$

where $K$ takes the values 1 and $1 / 2$ for $\Delta=0$ (free field), and $\Delta=1$ (isotropic anti-ferromagnet), respectively. The relation between $K$ and $\Delta$ is not preserved under the renormalization, so this relation is only correct for the initial Hamiltonian. The analytical form of the spin operators in 
terms of the bosonic fields are:

$$
\begin{aligned}
S_{n}^{x} & =\left[c_{2} \cos (2 \sqrt{\pi K} \phi)+(-1)^{n} c_{3}\right] \cos \left(\sqrt{\frac{\pi}{K}} \theta\right), \\
S_{n}^{y} & =-\left[c_{2} \cos (2 \sqrt{\pi K} \phi)+(-1)^{n} c_{3}\right] \sin \left(\sqrt{\frac{\pi}{K}} \theta\right), \\
S_{n}^{z} & =\sqrt{\frac{\pi}{K}} \partial_{x} \phi+(-1)^{n} c_{1} \cos (2 \sqrt{\pi K} \phi)
\end{aligned}
$$

where $c_{i}$ 's are constants as given in Ref. [27]. The Hamiltonian $H_{2}$ in terms of bosonic fields is the following,

$$
\begin{aligned}
H_{2}= & H_{0}+\frac{a}{2 \pi \alpha} \int \cos \left(2 \sqrt{\frac{\pi}{K}} \theta(x)\right) d x \\
& +\frac{\Delta}{2 \pi \alpha^{2}} \int \cos (4 \sqrt{\pi K} \phi(x)) d x+\frac{h \sqrt{K}}{\pi \alpha} \int \partial_{x} \phi(x) d x
\end{aligned}
$$

One can also write the above Hamiltonian in the following form

$$
\begin{aligned}
H_{2}= & H_{01}+\frac{a}{2 \pi \alpha} \int \cos (2 \sqrt{\pi} \theta(x)) d x \\
& +\frac{\Delta}{2 \pi \alpha^{2}} \int \cos (4 \sqrt{\pi} \phi(x)) d x+\frac{h}{\pi \alpha} \int \partial_{x} \phi(x) d x
\end{aligned}
$$

Where $H_{01}$,

$$
H_{01}=\frac{1}{2 \pi} \int d x\left[u K(\nabla \theta(x))^{2}+(u / K)(\nabla \phi(x))^{2}\right]
$$

One can get the $H_{X Y}$ Hamiltonian by simply putting $\Delta=0$ in the above Hamiltonian. In this derivation, different powers of coefficients $c_{i}$ have been absorbed in the definition of $a, h$ and $\Delta$. The integration of the oscillatory terms in the Hamiltonian yield negligible small contributions and the origin of the oscillatory terms occur due the spin operators. So it's a reasonably good approximation to keep only the non-oscillatory terms in the Hamiltonian. The Gaussian scaling dimension of these coupling terms, $a$ and $\Delta$ are $1 / K$ and $4 K$ respectively. The third term $(\Delta)$ of the Hamiltonian tends to order the system into density wave phase, whereas the second term $(a)$ of the Hamiltonian favors the staggered order in the $X Y$ plane. Two sine-Gordon coupling terms are from two dual fields. Therefore, the model Hamiltonian consists of two competing interactions. This Hamiltonian contains two strongly relevant and mutually nonlocal perturbation 
over the Gaussian (critical) theory. In such a situation the strong coupling fixed point is usually determined by the most relevant perturbation whose amplitude grows up according to its Gaussian scaling dimensions and it is not much affected by the less relevant coupling terms. However, this is not the general rule if the two operators exclude each other, i.e., if the field configurations which minimize one perturbation term do not minimize the other. In this case interplay between the two competing relevant operators can produce a novel quantum phase transition through a critical point or a critical line. Therefore, we would like to study the RG equation to interpret the quantum phases of the system. In the RG theory, we not only able to predict the weak coupling limit but also the the intermediate values of the coupling. The RG theory is a perturbative theory and it ceases to be valid when the coupling constant $g(l) \sim 1$.

We now study how the parameters $a, \Delta$ and $K$ flow under RG. The operators in Eq. (17) are related to each other through the operator product expansion. So the RG equations for their coefficients therefore are coupled to each other. We use operator product expansion to derive these RG equations which is independent of boundary condition [28]. In our derivation, we consider two operators, $X_{1}=e^{\left(i a_{1} \phi+i b_{1} \theta\right)}$ and $X_{2}=e^{\left(i a_{2} \phi+i b_{2} \theta\right)}$. In the RG procedure, one can write these two field operators as a sum of fast and slow mode fields. In the fast field, the momentum range is $\Lambda e^{-d l}<K<\Lambda$ and for the slow field $K<\Lambda e^{-d l}$, where $\Lambda$ is the momentum cut-off, $d l$ is the change in the logarithmic scale. The next step is the integration of the fast field for the operators $X_{1}$ and $X_{2}$, it yields a third operator at the same space time point, $X_{3}=e^{\left.i\left(a_{1}+a_{2}\right) \phi+i\left(b_{1}+b_{2}\right) \theta\right)}$. The prefactor of $X_{3}$ can be found by the relation, $X_{1} X_{2} \sim e^{-\left(a_{1} a_{2}+b_{1} b_{2}\right)} \frac{d l}{2 \pi} X_{3}$. Our Hamiltonian consists of two operators, if we consider $l_{1}$ and $l_{2}$ as the coefficient of the operators $X_{1}$ and $X_{2}$ respectively. Then the RG expressions for $\frac{d X_{3}}{d l}$ contains the term $\left(a_{1} a_{2}+b_{1} b_{2}\right) \frac{l_{1} l_{2}}{2 \pi}$. This is the procedure to derive these RG equations.

In the RG process, one can write RG equations themselves are established in a perturbative expansion in coupling constant $(g(l))$. They cease to be valid beyond a certain length scale, where $g(l) \sim 1[20]$. The RG equations for the coefficients of Hamiltonian $H_{X Y Z}$ are

$$
\frac{d a}{d l}=\left(2-\frac{1}{K}\right) a, \quad \frac{d \Delta}{d l}=(2-4 K) \Delta, \quad \frac{d K}{d l}=\frac{a^{2}}{4}-K^{2} \Delta^{2},
$$

We have followed Ref. [25] during the derivation of these RG equations. These RG equations have trivial $\left(a^{*}=0=\Delta^{*}\right)$ fixed points for any arbitrary $K$. Apart from that these RG equations have also two non-trivial fixed lines, 
$a=\Delta$ and $a=-\Delta$ for $K=1 / 2$. The above RG equations show that there is no duality in flow diagram. Here we mean, duality, that if $\theta$ and $\phi$ interchange $\theta \leftrightarrow \phi, K \leftrightarrow K^{-1}$ and $\Delta \leftrightarrow a$ will not produce the same set of RG equations.

For $K>1 / 2$, the sine-Gordon coupling term correspond to the anisotropic exchange coupling become relevant and the system flows to the photonic superfluid phase. For $K<1 / 2$, the sine-Gordon coupling term correspond to the $\mathrm{z}$-component exchange interaction become relevant and the system flowing off to the photon blocked induced insulating phase.

Here we explain the physical significance of different quantum phases of the atom-cavity system what we find in our study.

1) When both the anisotropic exchange interaction and the z-component of exchange interaction is absent then there are no sine-Gordon coupling terms in the Hamiltonian. Then the system is in the mass less Luttinger liquid phase, i.e., the system is in the photonic metallic state. The other source of photonic metallic state is that when the system shows the BKT transition which we will discuss in the next section.

2) The photonic insulator state of the atom-cavity array system corresponds to the insulating state of the system where there is no transmission of photon between the microcavities in the array due to the interaction between photons. The appearance of this phase has already discussed in the analysis of three RG equations. This phase will occurs when the system shows the BKT transition, which we will discuss in the next section.

3) Photonic superfluid state of the atom-cavity array system corresponds to the gapless excitations of the system where the photon transmit from one cavity to the other without any blocking. For this one dimensional cavity QED system where there is no order parameter, one can only discriminate between the photonic metallic state and photonic superfluid state by only finding the difference of fluctuation in photon number in every sites of the array. This photonic superfluid phase corresponds to dissipitionless flow of photon in the system. We have already discussed about the appearance of photonic superfluid phase from the analysis of three RG equations. The other source of the appearance of photonic superfluid phase is the BKT transition which we will discuss in the next section. 


\section{Berezinskii-Kosterlitz-Transition physics in cavity QED system}

The physics of BKT transition has found in different one dimensional and two dimensional (classical system) and it has discussed extensively in different context in the Ref. [18, 20, 21].

Before we start to discuss the appearance of BKT transition in our system, we would like to discuss very briefly why it is necessary to study the BKT transition. Here we study two different situations of our model Hamiltonian. For the first case the exchange anisotropy is absent $\left(J_{2}=0\right)$ and for the second case z-component of exchange interaction is absent $\left(J_{z}=\right.$ $0)$. For both of these cases only one of the sine-Gordon coupling term is present, therefore, there is no competition between the two mutually non local perturbation. Therefore one can think that there is no need to study the RG to extract the quantum phases and phase boundaries. But we still apply RG method for the following reason. Each of these Hamiltonians consist of two part, the first one $\left(H_{01}\right)$ is the non-interacting where the $\phi$ and $\theta$ fields show the quadratic fluctuations and the other part of these Hamiltonians are the sine-Gordon coupling terms which of either $\theta$ or $\phi$ fields. The sine-Gordon coupling term lock the field either $\theta$ or $\phi$ in the minima of the potential well. Therefore the system has a competition between the quadratic part of the Hamiltonian and the sine-Gordon coupling term and this competition will govern the low energy physics of these Hamiltonians in different limit of the system. The RG process (BKT transition) will predict the quantum phases of these system correctly.

It is very clear from the analytical expression of $J_{1}, J_{2}$ and $J_{z}$ that one can control these parameters in the laboratory. The quantum state of engineering of cavity QED system is in the state of art due to the rapid technological development of this system [1]. In this study we consider the situations where the $J_{2}$ is absent, i.e., the system with a single Rabi frequency oscillation. We also consider the situation where the z-component of exchange interaction is absent. These two situations lead to the two different set of RG equations which show BKT transition.

For the first case, there is no anisotropic exchange coupling. In this situation, the three $\mathrm{RG}$ equations of the previous section reduce to two $\mathrm{RG}$ equations, which are the following

$$
\frac{d \Delta}{d l}=(2-4 K) \Delta, \quad \frac{d K}{d l}=-K^{2} \Delta^{2},
$$

Now we express these RG equations in the form of BKT transition form. As 
we understand from the RG equations that the transition occurs at $K=1 / 2$. To study the flow of the RG equation around this transition point, we recast the $\mathrm{RG}$ equation in suitable form.

Here we follow the following transformation, $K=1 / 2+\frac{y_{\|}}{4}$ and $\Delta \rightarrow$ $\Delta / 2$. The above equation reduce to the standard BKT equation.

$$
\frac{d y_{\|}}{d l}=-\Delta^{2}, \quad \frac{d \Delta}{d l}=-y_{\|} \Delta
$$

In our case, $\Delta \frac{d \Delta}{d l}=y_{\|} \frac{d y_{\|}}{d l}$. Therefore $X^{2}=y_{\|}^{2}-\Delta^{2}$ is a constant of motion. Here we discuss the relevant physics and the quantum phase transition between the photonic metallic state and photonic Coulomb blocked state based on these equations. Here we consider the following situations based on these equations:

1). When $X>0$ and $y_{\|}>0$, the sine-Gordon coupling term corresponding to $\Delta$ term is irrelevant, the fixed point, $\Delta^{*}=0$ and $y_{\|}{ }^{*}=X$, close to this fixed point, we can write the RG equations as $\frac{d \Delta}{d l}=2\left(1-2 K^{*}\right) \Delta(l)$ and $\frac{d y_{\|}}{d l}=0$. Using the flow equation and constant of motion for $y_{\|}>\Delta$. One can write the solution of coupling terms as

$$
\begin{aligned}
y_{\|}(l) & =\frac{X}{\tanh \left(X l+\operatorname{atanh}\left(\frac{X}{y_{\|}{ }^{0}}\right)\right.} \\
\Delta(l) & =\frac{X}{\sin \left(X l+\operatorname{atanh}\left(\frac{X}{y_{\|}{ }^{0}}\right)\right.}
\end{aligned}
$$

The condition at the line of seperatix is $y_{\|}(l)=\Delta(l)=\frac{\Delta^{0}}{1+\Delta^{0} l}$. There is no mass gap excitation in the elementary excitation of the system, i.e, there is no photon blocked induced insulating phase. Hence the system is in the photonic metallic state.

To the best of our knowledge for the first time in the literature we predict the existence of photonic metallic state for the array of Cavity QED system. Here we would like to present the basic origin of photonic-metallic state explicitly.

If we do the Jordan-Wigner transformation of our model Hamiltonian as we present in Ref. [22]. It is very clear from the Eq. 32 of Ref. [22] that the first term represent the photon hopping term across the lattice of the cavity QED array. The second and third term of the Hamiltonian represent the photonic pair correlation and photonic density wave respectively in the system. When the second and third term in the Hamiltonian either are 
absent or irrelevant in the RG sense at that situation the system shows the photonic metallic state.

2). When $\Delta>y_{\|}$. The RG equation for the coupling $\Delta(l)$ flowing off to the strong coupling regime. A perturbative expansion in $\Delta$ cease to be valid beyond a certain length scale for which $\Delta(l) \sim 1$. The analysis of the RG equation is not valid beyond this length scale.

The analytical relation between the coupling constant is

$$
\arctan \left(\frac{y_{\|}^{0}}{\sqrt{\Delta_{0}^{2}-y_{\|} 0^{2}}}\right)-\arctan \left(\frac{y_{\|}}{\sqrt{\Delta_{0}^{2}-y_{\|} 0^{2}}}\right)=\sqrt{\Delta_{0}^{2}-y_{\|}^{0^{2}}}
$$

In this limit, the RG flowing off to the strong coupling phase. Here we discuss the relevant physics of the phase. The sine-Gordon coupling term is

$$
\frac{\Delta_{1} u}{2 \pi \alpha^{2}} \int d x \cos (4 \phi(x))
$$

where $\Delta_{1}=\frac{\Delta}{\pi u}$. The $\phi(x)$ field locks into one of the minima of the cosine potential. Now we expand the potential for large $\Delta_{1}$ in the spirit of usual RG method [18, 20, 21]. We can write the effective Hamiltonian near to the minima

$$
H=H_{0}+\frac{4 \Delta_{1} u}{\pi \alpha^{2}} \phi^{2}(x) .
$$

The total action of the Hamiltonian of the system can be written as

$$
S=\frac{1}{2 \pi K} \frac{1}{\beta \Omega} \sum_{k, \omega_{n}}\left[\frac{\omega_{n}^{2}}{u}+u k^{2}+\frac{8 K \Delta_{1} u}{\alpha^{2}}\right] \phi^{*}\left(k, \omega_{n}\right) \phi\left(k, \omega_{n}\right)
$$

The excitations of the system which cost a finite energy even at $k=0$. In this situation the field, $\phi(x)$ is massive. The system posses phononic type mode. This phononic mode is the small oscillation of the field $\phi(x)$ around the minima of the cosine potential.

Suppose we consider the RG equation up to the point where $\Delta_{1}(l) \sim 1$. This excitations gap in the spectrum has the dimension of an energy and the renormalization relation is $\Omega_{M}(l)=e^{l} \Omega_{M}(l=0)$, for the case, $\Delta_{1}\left(l^{*}\right) \sim 1$. We can write the expression for the gap following the action as, $\Omega_{M}\left(l^{*}\right) \sim$ $\sqrt{\Delta_{1}\left(l^{*}\right)} u / \alpha$. The true gap of the system is $\Omega_{M}(l=0) \sim e^{-l^{*}} \Omega_{M}\left(l^{*}\right)$, $\Omega_{M}\left(l^{*}\right)=\frac{u}{\alpha}$. Thus at this phase of this system, the explicit dependence of the Luttinger liquid parameter is absent. This gap of the system is of the order of bandwidth of the cavity QED system. 
3). Now we consider the case, when $\Delta_{1} \ll\left|y_{\mid}\right|$. The system is in the deep massive phase. In this phase, one can write the RG equation as $\frac{d K(l)}{d l}=0$ and $\frac{d \Delta_{1}(l)}{d l}=2(1-2 K) \Delta_{1}(l)$. For this RG equation, we get $\Delta_{1}(l)=\Delta_{1}(0)$ $\cdot e^{2(1-2 K) l}$ from this equation $e^{-l^{*}}=\Delta(0)^{1 / 2(1-2 K)}$. The true gap of the system is $\Omega_{2}(l=0) \sim e^{-l^{*}} \Omega_{2}\left(l^{*}\right), \frac{\Omega_{2}(l=0)}{\Omega_{2}\left(l^{*}\right)} \sim \Delta_{1}(0)^{+1 / 2(1-2 K)}$. This gap is the power law dependence of the bare $\Delta_{1}$. This gap varies with $K$, i.e., the gap of the system is now varying with the interaction of the system. It is very clear from the analytical expression that the gap gets smaller and tends to zero at $K=1 / 2$, this prediction is consistent with the physical scenario that at $K=1 / 2$ the system shows the phase transition.

4). Close to the transition point, Eq. (25) reduces to $\sqrt{\Delta_{10}^{2}-\left(y_{\|}\right)^{2}} l^{*}=\pi$. So the square root term goes to zero at the transition point and therefore

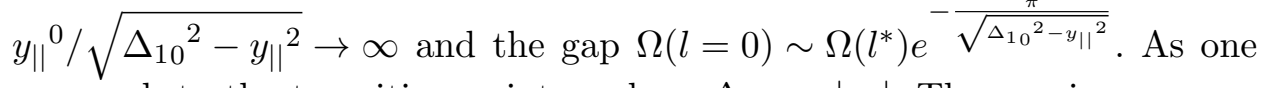
approach to the transition point, such as $\Delta_{10} \rightarrow\left|y_{||}\right|$. The gap is exponentially small in the square root of the distance to the transition.

It is therefore clear from our RG analysis that the system is in the massive phase for the two limits. For the first case when $\Delta \gg y_{\|}$, the excitation gap of the system is of the order of the bandwidth of the system and the effect of photonic strong correlation is not explicit. The other limit of gapped state, i.e, when $\Delta \ll\left|y_{||}\right|$. The excitation gap of the system has a power law dependence which vary with $K$.

It is very clear from the analytical expression of $J_{z}$ that one can tune it to zero by adjusting the Rabi frequencies oscillation of the system. In this situation, there is no z-component of intercavity exchange interaction. Therefore the three RG equations are reduce to two RG equations.

$$
\frac{d a}{d l}=(2-1 / K) a, \quad \frac{d \ln K}{d l}=a^{2} / 4 K,
$$

If we do the following transformation $\tilde{K}=1 / 2 K$ and $\tilde{a}=a / 2$ and after that we follow another transformations $\tilde{K}=1+y_{\|} / 2$ the above equations reduce to

$$
\frac{d y_{\|}}{d l}=-\tilde{a}^{2}, \quad \frac{d \tilde{a}}{d l}=-y_{\|} \tilde{a}
$$

The mathematical structure of these equations are the same as that of Eq. 22. Therefore the mathematical analysis for the different limits are the same but massive phase and deep massive phase for these situations are the 
photonic superfluid phase.

The sine-Gordon coupling term is

$$
\frac{a_{2} u}{2 \pi \alpha} \int d x \cos (2 \theta(x)) .
$$

where $a_{2}=\frac{a}{u}$. The $\theta(x)$ field locks into one of the minima of the cosine potential. Now we expand the potential for large $a_{2}$. We can write the effective Hamiltonian near to the minima

$$
H=H_{0}+\frac{a_{2} u}{\pi \alpha} \theta^{2}(x) .
$$

The total action of the Hamiltonian of the system can be written as

$$
S=\frac{1}{2 \pi K} \frac{1}{\beta \Omega} \sum_{k, \omega_{n}}\left[\frac{\omega_{n}^{2}}{u}+u k^{2}+\frac{2 K a_{2} u}{\alpha}\right] \theta^{*}\left(k, \omega_{n}\right) \theta\left(k, \omega_{n}\right)
$$

The excitations of the system which cost a finite energy even at $k=0$. In this situation the field, $\theta(x)$ is massive. The true gap of the system is $\Omega_{C}(l=0) \sim e^{-l^{*}} \Omega_{C}\left(l^{*}\right), \Omega_{C}\left(l^{*}\right)=\left(\frac{u}{\alpha}\right) \sqrt{\alpha}$. Thus at this phase of this system, the explicit dependence of the Luttinger liquid parameter is absent. This gap of the system is much less than the excitation gap of the photon blocked state due to the presence of the extra factor $\sqrt{\alpha}$.

In the deep massive phase, $\frac{\Omega_{C}(l=0)}{\Omega_{C}\left(l^{*}\right)} \sim a_{2}(0)^{+1 /(2-1 / K)}$. This gap is the power law dependence of the bare $a_{2}$. This gap varies with $K$, i.e., the gap of the system is now varying with the interaction of the system. It is very clear from the analytical expression that the gap gets smaller and tends to zero at $K=1 / 2$ and this prediction is consistent with the physical scenario that at $K=1 / 2$ the system shows the phase transition from photonic metallic state to photonic superfluid phase.

Now we consider the effect of magnetic field ( $\delta_{1}$ term) on the quantum phases and phase boundaries of this cavity QED arrays.

We do the following transformation to eliminate the magnetic field term from the Hamiltonian. We substitute $4 \pi \sqrt{K} \phi \rightarrow 4 \pi \sqrt{K} \phi+4 \pi \delta x$, where $\delta=$ $\sqrt{K} h$. This introduce the spatial oscillation in the $\Delta$ term. When the coupling $a$ is absent and the applied detuning field is larger than the photonic Mott gap, the system drives to the gapless photonic metallic state. This is nothing but the well known Porkovosky-Talapov model which shows the commensurate to incommensurate transition $[20,25]$. When $\delta a_{1} \gg 1$ (where $a_{1}$ is the lattice spacing), the $\cos (4 \pi \sqrt{K}(\phi+\delta x))$ term is quickly oscillating and averages out zero. Thus the system reflects the competition between 
the $\Delta$ and $h$. As a results of it, the RG flow for $\Delta$ has to be cutoff when $4 \pi \sqrt{K} \delta(l) a_{1} \sim 1$. To the lowest order in $\Delta, a$ and $\delta$, the RG flow equation is

$$
\frac{d \delta}{d l}=\delta
$$

As a result of this RG equation, the system shows two different response, either the flow $\Delta_{1}$ and $\delta$ or $a$ and $\delta$ flows of to the strong coupling phase.

When $\Delta_{1}(l)$ reaches strong coupling before $\delta a_{1}$ become order one. The phase boundary is the same as we predict without magnetic field. The condition when $\Delta_{1}\left(l^{*}\right) \sim 1$ define a new length scale $l^{*}$. It generates a selfconsistent scenario that $4 \pi \delta\left(l^{*}\right) a_{1} \ll \Delta_{1}(0)^{1 /(2-4 K)}$. In the other limit when the RG flow of $a$ flows off to the strong coupling phase does not affected by the magnetic field term and thus the system is in the photonic superfluid phase.

\section{Conclusions}

We have presented three sets of RG equations for the different physical situations for the Cavity QED system. We have predicted two different BKT transitions for the different physical situations. We have predicted the photonic superfluid phase, photonic metallic phase and photon blocked induced insulating phase. To the best of our knowledge, this explicit quantum phase analysis and their behavior based on the RG study for the microcavity array system is absent in the previous literature.

\section{Acknowledgments}

The author would like to acknowledge the DST fund, the Library of Harish Chandra Research Institute, Raman Research Institute, the series of lectures and also discussions of Prof. S. M. Girvin during the International Workshop/School on Dirac Materials and Chandrashekar Discussion Meeting, December'2012. The author would like to thank The Center for Condensed Matter Theory of the Physics Department of IISc for extended facility. Finally the author would like to thank Prof. Prabir Mukherjee for reading this manuscript critically. 


\section{References}

[1] G. S. Agarwal, Quantum Optics Cambridge University Press, Cambridge (2013).

[2] S. M. Girvin, Circuit QED: Superconducting Qubits Coupled to Microwave Photons, Lecture Notes/Private Communication.

[3] Michael J. Hartmann, Fernando G. S. L. Brandão and Martin B. Plenio, Strongly interacting polaritons in coupled arrays of cavities, Nature Phys 462 (2006) 849; Laser and Photonics Rev. 2 (2008) 527.

[4] Michael J. Hartmann, Fernando G. S. L. Brandão and Martin B. Plenio, Effective Spin Systems in Coupled Microcavities, Phys. Rev. Lett 99 (2007) 160501.

[5] S. Sarkar, Quantum field theoretical study of an effective spin model in coupled optical cavity arrays, Physica B 407, 44 (2012).

[6] S. Horoche and J. M. Raimond, Exploring the Quantum Atoms, Cavities, and Photons, Oxford University Press, (2006).

[7] E. T. Jaynes and F. W. Cummings, Comparison of quantum and semiclassical radiation theories with application to the beam maser, Proc. IEEE 51, 89 (1963).

[8] A. C. Ji, X. C. Xie and W. M. Liu, Quantum Magnetic Dynamics of Polarized Light in Arrays of Microcavities, Phys. Rev. Lett. 99, 183602 (2007).

[9] T. Byrnes, N. Y. Kim, K. Kusudo and Y. Yamamoto, Quantum simulation of Fermi-Hubbard models in semiconductor quantum-dot arrays, Phys. Rev. B 78075320 (2008).

[10] I. Carusotto et al., Fermionized photons in an array of driven dissipative nonlinear cavities, arXiv:0812.4195.

[11] M. J. Bhaseen et al., Polaritons and Pairing Phenomena in BoseHubbard Mixtures, Phys. Rev. Lett. 102135301 (2009).

[12] J. Zhao, A. W. Sandvik and K. Ueda, Insulator to superfluid transition in coupled photonic cavities in two dimensions, arXiv:0806.3603.

[13] P. Pippan, H. G. Evertz and M. Hohenadler, Excitation spectra of strongly correlated lattice bosons and polaritons, arXiv:0904.1350. 
[14] M. Aichhorn et al., Quantum Fluctuations, Temperature, and Detuning Effects in Solid-Light Systems, Phys. Rev. Lett. 100216401 (2008).

[15] S. Schmidt and G. Blatter, Strong coupling theory for the JaynesCummings-Hubbard model, arXiv:0905.3344.

[16] D. G. Angelakis, M. F. Santos and S. Bose, Photon-blockade-induced Mott transitions and XY spin models in coupled cavity arrays, Phys. Rev. A 76 R031805, (2007).

[17] B. Kumar, Emergent radiation in an atomfield system at twice resonance, J. Phys. A: Math. Theor 42, 245307 (2009);

[18] Sachdev Subir, Quantum Phase Transition, Cambridge University Press, Cambridge, (1998).

[19] A. K. Pal and I. Bose, Quantum discord in the ground and thermal states of spin clusters, J. Phys. B: At. Mol. Opt 44, 045101 (2011).

[20] T. Giamarchi, Quantum Physics in One Dimension, Clarendon Press, Oxford, (2004).

[21] Itzykson and Drouffe, Statistical Field Theory, Volume 1, Cambridge University Press, New York (1989).

[22] Here we present the Jordon-Wigner transformation of Eq. 15, $Q_{i}=$ $e^{i \pi \sum_{n=1}^{i-1} c_{i}^{\dagger} c_{i}}, S_{i}^{-}=Q_{i} c_{i}, S_{i}^{+}=c_{i}^{\dagger} Q_{i}^{\dagger}$. The Eq. 15 under this trnsformation become

$$
\begin{aligned}
H= & \sum_{i=1}^{n}\left(c_{i+1}{ }^{\dagger} c_{i}+h . c\right)+a\left(c_{i}^{\dagger} c_{i+1}^{\dagger}+h . c\right) \\
& +\Delta \sum_{i}\left(c_{i}^{\dagger} c_{i}-1 / 2\right)\left(c_{i+1}^{\dagger} c_{i+1}-1 / 2\right)+h\left(c_{i}^{\dagger} c_{i}-1 / 2\right)
\end{aligned}
$$

It is clear from the above expression that the anisotropic-exchange interaction induce creation or annihilation of photon pairs.

[23] K. M. Birnbaum et al., Photon blockade in an optical cavity with one trapped atom, Nature 436, 87 (2005).

[24] F. V. James and J. Jerke, Effective Hamiltonian theory and its applications in quantum information, Can. J. Phys 85, 625 (2007).

[25] S. Sarkar, Critical and off-critical properties of an anisotropic Heisenberg spin- $\frac{1}{2}$ chain under a transverse magnetic field, Phys. Rev. B 74, 052410 (2006). 
[26] U. Schollwock, J. Richter, D. J. J. Farnell and R. F. Bishop eds., Quantum Magnetism, Lect. Notes Phys. 645, Springer, Berlin, (2004).

[27] S. Lukyanov and A. Zamolodchikov, Exact expectation values of local fields in the quantum sine-Gordon model, Nucl. Phys. B 493, 571 (1997).

[28] J. Cardy, Scaling and Renormalization in Statistical Physics, Cambridge University Press, Cambridge, (1996); I. Affleck, Fields, Strings and and Critical Phenomena, E. Brezin and J. Zinn-Justin eds., North-Holland, Amstardam, (1989).

Poornaprajna Institute of Scientific Research

4 Sadashivanagar, Bangalore 5600 80, India.

E-mail address: sujit.tifr@gmail.com 